medgen $2018 \cdot 30: 229-230$

https://doi.org/10.1007/s11825-018-0195-1

Online publiziert: 27. Juni 2018

(c) Springer Medizin Verlag GmbH, ein Teil von Springer Nature 2018

CrossMark

Alexander E. Volk · Christian Kubisch

Institut für Humangenetik, Universitätsklinikum Hamburg-Eppendorf, Hamburg, Deutschland

\title{
Neurodegenerative Erkrankungen
}

Der Begriff neurodegenerative Erkrankungen ist ein Sammelbegriff für klinisch z.T. sehr unterschiedliche Erkrankungen, bei denen - zumindest initial Nervenzellen des Gehirns oder spezieller Regionen des Gehirns geschädigt werden und im weiteren Verlauf zugrunde gehen. Dies führt in aller Regel zu zunehmenden und irreversiblen motorischen, sensiblen und/oder kognitiven Symptomen. Ein Themenheft der medizinischen genetik speziell zu neurodegenerativen Erkrankungen gab es in dieser Form noch nicht, auch wenn einzelne Themen und Erkrankungen bereits in anderen Schwerpunktheften aufgegriffen wurden. Aufgrund der Vielzahl von neuen Erkenntnissen zur Genetik, Pathophysiologie und Klinik dieser Erkrankungsgruppe in den letzten Jahren, erschien es aus unserer Sicht jetzt jedoch sinnvoll, einen eigenständigen Themenschwerpunkt Neurodegeneration heraus zu geben.

Die meisten neurodegenerativen Erkrankungen sind mit dem Alter assoziiert und stellen eine alternde Gesellschaft vor medizinische, sozioökonomische und ethische Herausforderungen. Schätzungen zufolge wird aufgrund des demographischen Wandels die Gesamtzahl von Patienten mit einer neurodegenerativen Erkrankung in Deutschland von heute etwa zwei Millionen auf drei Millionen im Jahr 2050 ansteigen. Genetische Risikofaktoren sind praktisch bei allen neurodegenerativen Erkrankungen bekannt, wobei deren Bedeutung für $\mathrm{Di}$ agnostik und Familienberatung je nach Krankheitsentität variieren. Eine besondere Herausforderung ist auch dadurch gegeben, dass bei vielen neurodegenerativen Erkrankungen sowohl monogene als auch multifaktorielle Formen exis- tieren, ohne dass beide Gruppen sich klinisch klar unterscheiden. Tatsächlich konnte gezeigt werden, dass komplexgenetische Risikofaktoren (identifiziert durch genomweite Assoziationsstudien) und monogene Krankheitsgene bei einigen neurodegenerativen Erkrankungen dieselben genetischen Loci bzw. Gene betreffen, was die Überlappung der Krankheitsformen untermauert und was in einzelnen Artikeln dieses Heftes weiter ausgeführt wird.

Die beiden häufigsten neurodegenerativen Erkrankungen, d.h. die Alzheimer-Demenz und der M. Parkinson, werden in zwei separaten Beiträgen mit Schwerpunkt auf die selteneren monogenen Subtypen der Erkrankungen behandelt. Im Gegensatz dazu ist bei der genetisch sehr heterogenen Gruppe der hereditären spastischen Paraparesen der Anteil an monogenen Formen sehr hoch. Im entsprechenden Artikel wird dargestellt, wie v.a. Hochdurchsatz-Sequenzieransätze dabei geholfen haben, neue Krankheitsgene zu identifizieren, so dass inzwischen hohe diagnostische Aufklärungsraten erreicht werden können. Komplementiert wird dieser Fachartikel durch eine kurze Vorstellung der Tom-Wahlig-Stiftung, die seit nunmehr 20 Jahren die Forschung im Bereich der spastischen Paraparesen fördert.

Neben den hereditären spastischen Paraparesen ist die amyotrophe Lateralsklerose eine weitere neurodegenerative Erkrankung mit Motoneuronbeteiligung. Unbestritten hat in den letzten Jahren die öffentliche Wahrnehmung der ALS durch die ice bucket challenge und zuletzt durch den Tod Stephen Hawkins zugenommen. Die genetischen Aufklärungsraten sind zwar geringer als bei der HSP, jedoch wurden auch hier in 
den letzten Jahren neue krankheitsrelevante Gene identifiziert, die gemeinsam mit einem internationalen Koautor in einem englischsprachigen Artikel zusammengefasst werden.

In einem Themenheft zu neurodegenerativen Erkrankungen darf die Huntington-Krankheit natürlich nicht fehlen. Das 25-jährige Jubiläum der Genidentifikation und die Geschichte dahinter wären allein einen Beitrag wert. Neben den genetischen Aspekten beschreibt der Beitrag aktuelle Therapieansätze und Ergebnisse der ersten klinischen Studien bei der Chorea Huntington.

Entgegen der weitläufigen Meinung, dass neurodegenerative Erkrankungen nur im Alter auftreten, werden in diesem Heft auch neurodegenerative Erkrankungen des Kindesalters dargestellt. Exemplarisch wird dabei die Gruppe der neuronalen Ceroid Lipofuszinosen (NCL) beschrieben, wobei insbesondere innovative therapeutische Konzepte für diese „seltene“ Erkrankung zum Thema gemacht werden. Auch dieser Fachbeitrag wird komplementiert durch eine kurze Darstellung der NCL-Stiftung, die neben der spezifischen Forschungsförderung im Bereich NCL auch allgemeine Fragestellungen und Herausforderungen bei seltenen Erkrankungen in den Fokus stellt.

Wir danken allen Autoren für Ihre Beiträge und hoffen, dass wir mit diesem Themenheft einerseits einen aktuellen Überblick zu den jeweiligen genetischen Hintergründen und Möglichkeiten der genetischen Diagnostik und anderseits tiefere Einblicke zum aktuellen Forschungsstand sowie (zukünftigen) therapeutischen Konzepten der neurodegenerativen Erkrankungen geben können.

\section{Korrespondenzadresse}

\section{Prof. Dr. med. C. Kubisch}

Institut für Humangenetik, Universitätsklinikum Hamburg-Eppendorf

Martinistr. 52, 20246 Hamburg, Deutschland

c.kubisch@uke.de

Interessenkonflikt. A.E. Volk und C. Kubisch geben an, dass kein Interessenkonflikt besteht. 